\title{
Unterstützung des Variantenmanagements von Elektromotoren durch wissensbasierte Systeme
}

Johann Tüchsen ${ }^{1,2}$, Julian Dorsch², Moritz Obendorfer², Benjamin Schleich ${ }^{1}$, Sandro Wartzack ${ }^{1}$

${ }^{1}$ Engineering Design (KTmfk), Friedrich-Alexander-Universität Erlangen-Nürnberg (FAU), Germany

2 Brose Fahrzeugteile GmbH \& Co. KG, Würzburg, Germany

\section{Abstract}

The automotive industry electrifies a huge variety of previously mechanically-actuated car functions. While sharing the same function, the used electric motors often differ in customer related geometries and interfaces. The suppliers have to cope with this huge external variance using an appropriate variant management. With this paper, the support of the variant management of electric drives by knowledge-based systems is proposed. Therefore, a combination of two systems containing the knowledge of the configuration and design of motors are used. The concept is applied and evaluated on the example of the shaft rotor lamination connection. As a result, the amount of internal variants can be reduced and controlled as well as the designer can be supported during the development process.

Keywords: Variant Management, Knowledge-based Systems, KBS, Electric Drives 


\section{Einleitung und Motivation}

Durch die Bestrebungen der Automobilindustrie, Fahrzeuge zunehmend zu elektrifizieren, hält neben elektrischen Traktionskonzepten auch eine Vielzahl an kleineren Elektromotoren wie beispielsweise Lenkungsmotoren Einzug in moderne Personenkraftwägen. Einerseits lassen sich diese in großen Stückzahlen gefertigten Motoren basierend auf der gleichen Funktion vereinheitlichen. Andererseits benötigen aber die verschiedenen Applikationen unterschiedliche Motortopologien und -geometrien. Ein gängiges Motorkonzept ist der bürstenlose permanenterregte Synchronmotor, welcher wiederum eine Komponente des Systems Antrieb ist. Er besteht im Wesentlichen aus der Statorbaugruppe mit Wicklung und der Rotorbaugruppe mit Magneten und Welle (s. Bild 1). [1]

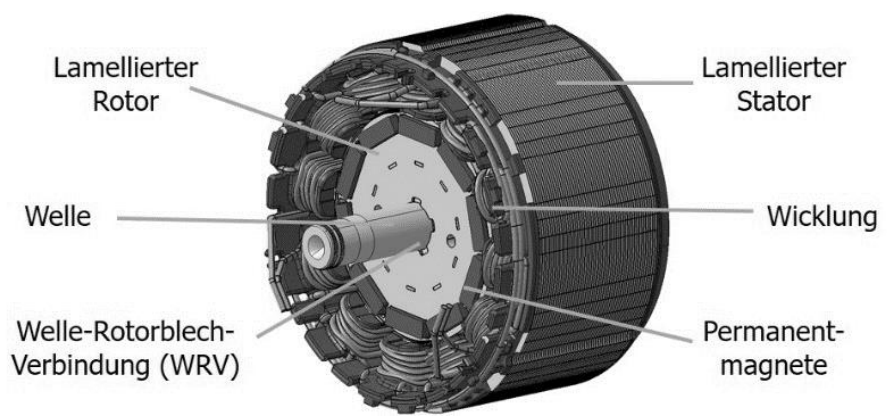

Bild 1: Prinzipieller Aufbau eines bürstenlosen Synchronmotors

Die reibschlüssigen Welle-Rotorblech-Verbindung (WRV) unterliegt sowohl äußeren Anforderungen des Kunden sowie internen Anforderungen der Elektromagnetik, Mechanik, Fertigung und Montage. Eine individuelle Auslegung dieser Schnittstelle für jedes Kundenprojekt benötigt große Mengen an Ressourcen und erzeugt eine hohe interne Varianz. Damit ein Unternehmen den Markt mit dieser Vielfalt versorgen und gleichzeitig den Material- und Entwicklungsaufwand geringhalten kann, bedarf es einem geeigneten Variantenmanagement.

Deshalb stellt sich folgende Forschungsfrage: Wie lassen sich die interne Variantenvielfalt und die Aufwände bei der Entwicklung von Elektromotoren reduzieren und beherrschen? Im Rahmen dieser Arbeit soll untersucht werden, ob die Forschungsfrage durch die Verwendung von modularen Produktstrukturstrategien in Kombination mit wissensbasierten Systemen beantwortet werden kann. Die Validierung des Ansatzes erfolgt am Bespiel der WRV. Die Auslegung von Welle-Nabe-Verbindungen (WNV) ist in der Literatur bereits umfassend bekannt [2]. Die abweichende Bezeichnung WRV statt WNV soll innerhalb 
dieses Beitrags den Anwendungsbezug zu den lamellierten Elektroblechpaketen hervorheben. Der Neuheitswert des Ansatzes liegt in der gezielten Kombination von Produkt-Konfiguration und -Auslegung in einem automatisierten, wissensbasierten Assistenzsystem für gekoppelte, mechatronische Schnittstellen.

Zunächst erfolgt ein Überblick über den Stand der Wissenschaft und Technik zum Variantenmanagement und der wissensbasierten Produktentwicklung (Abschnitt 2). Anschließend wird das neue Konzept zur wissensbasierten Unterstützung des Variantenmanagements für Elektromotoren vorgestellt (Abschnitt 3) und auf das Beispiel der WRV angewandt. Abschließend werden eine Zusammenfassung und ein Ausblick gegeben (Abschnitt 4).

\section{Stand der Wissenschaft und Technik}

\subsection{Variantenmanagement}

Ziel des Variantenmanagements ist es, die Produktvielfalt so zu wählen, dass ein Optimum zwischen Kosten und Nutzen entsteht [3]. Zu den Aufgaben zählen neben der Generierung von Varianten vor allem deren Vermeidung, Beherrschung und Reduzierung. Eine Reduzierung der internen Varianz und der damit verbundenen Komplexität ist unter anderem durch Nutzung von Kommunalitäten möglich [4]. Produktfamilien teilen dabei oft ihre Technologie und adressieren ähnliche Marktapplikationen [5]. Weiter hat jede Produktfamilie eine eigene Produktarchitektur, welche die Anordnung von Funktionselementen, deren Zuordnung zu physischen Komponenten und die Spezifikation der Schnittstellen zwischen interagierenden physischen Komponenten umfasst [6]. Die physische und hierarchische Zusammensetzung eines Produkts aus seinen Komponenten und deren Beziehungen wird als Produktstruktur definiert. Durch modulare Produktstrukturstrategien lässt sich innerhalb einer Produktfamilie eine Vielzahl verschiedener Varianten erzeugen. Zu diesen Strategien zählen die Plattform, Baukasten, Baureihen und die Wiederholmodul-Strategien, wobei diese im Unternehmen meist in Form einer Kombination Anwendung finden. Um ein Produkt durch modulare Produktstrukturstrategien abbilden zu können, muss zunächst eine Produktstrukturierung durgeführt werden. Dabei untergliedert sich das generische Vorgehen in die Schritte der Dekomposition der bestehenden Produktstruktur, der Analyse der Komponenten, der Bildung der Module sowie der Überführung in eine modulare Produktstruktur. [4]

Meyer und Lehnerd stellten fest, dass sich elektrische Motoren aufgrund ihres Aufbaus besonders gut für die Produktstrukturierung eignen [5]. So lassen sich ihre Eigenschaften (z.B. das Drehmoment) durch Längenskalierung und Anpassung der Wicklung bei einer fixierten 2D-Geometrie innerhalb sorgfältig 
definierter Grenzen skalieren und somit verschiedene Anforderungen erfüllen. Basierend auf diesen Eigenschaften entwickelten Roach und Cox einen webbasierten Produktkonfigurator für Elektromotoren [7]. Ponn stellte darüber hinaus das Variantenmanagement von Elektromotoren in einem Produktportfolio von Power-Tools vor [8]. Hierbei wird in der englischsprachigen Literatur oft von einer Plattform als Produktstrukturstrategie gesprochen [5,7], wohingegen in der deutschsprachigen Literatur bei Produkten, die bis auf wenige skalierbare Parameter identisch sind, eher von einer Baureihe die Rede ist (s. Bild 2, links). Bei der in den Arbeiten nicht betrachteten WRV kann ein Modulbaukasten verwendet werden, aus welchem durch Kombination von Modulen neue Produktvarianten erzeugt werden können (s. Bild 2, rechts). [4]
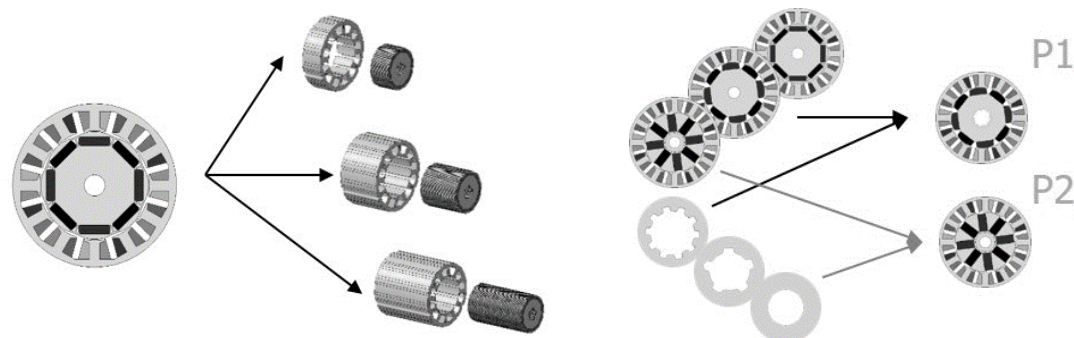

Bild 2: Modulare Produktstrukturstrategien Baureihe (links) und Modulbaukasten (rechts) am Beispiel Elektromotor

\subsection{Wissensbasierte Produktentwicklung}

Die Produktentwicklung zählt zu den wissensintensiven Prozessen, welche es durch ein geeignetes Wissensmanagement (WM) zu unterstützten gilt [9]. Dabei sind Planung, Identifikation, Bewertung, Erzeugung, Speicherung sowie die Verteilung und Anwendung Kernaktivitäten des WM [10]. Gerade in der Produktentwicklung liegt Wissen sowohl explizit in Form von Gleichungen als auch implizit als Erfahrung der Entwickler vor. Durch die Formalisierung des impliziten Wissens kann dieses in explizite Form transformiert werden [11] und lässt sich weiter in wissensbasierten Systemen (engl. Knowledge-based Systems, KBS) verarbeiten. Im Rahmen dieser Arbeit werden die Begriffe KBS und KBE (Knowledge-based Engineering, dt. wissensbasierten Konstruktion, [12]) synonym verwendet. Die Grundlegende Architektur eines KBS setzt sich aus den Komponenten User Interface, Inferenzmechanismus, Wissensdatenbank sowie den Wissenserwerb-, Erklärungs- und Kontextkomponenten zusammen [13]. Das Wissen wird durch Experten in die Wissensdatenbank eingepflegt und gewartet. Dazu stehen verschiedene Methoden der Wissensrepräsentation zur Verfügung. Zu nennen sind beispielsweise Ontologien, Logik, Unified Modelling 
Language [11] oder die objektorientierte Programmierung. KBS eignen sich besonders gut zur Unterstützung von Design- und Entscheidungsprozessen und rücken damit im Sinne der Design Automation in den Fokus [12].

Für die wissensbasierte Produktentwicklung elektrischer Motoren mit KBS existieren bereits verschiedene Ansätze. Favi et al. entwickelten die web-basierte Plattform EROD, um den Motorenentwickler bei der Konfiguration neuer Produkte hinsichtlich verschiedener Anforderungen zu unterstützen [14]. Karnavas and Chasiotis entwickelten ein KBS zur automatischen und regelbasierten Auslegung von Elektromotoren mit grundlegenden Berechnungen und Bewertungen [15]. Rivera et al. stellten ein Konzept zur Entwicklung elektrischer Maschinen mit KBS vor, welches auf den VDI-Normen 2221 und 2206 zur Produktentwicklung basiert [16]. Die genannten Konzepte fokussieren die Unterstützung der Entwicklung von Elektromotoren hinsichtlich Entscheidungs- und Designfindung, eine Betrachtung der Aspekte des Variantenmanagements erfolgte bisher jedoch nicht. Tüchsen et al. entwickelten einen Design Selector and Generator, ein KBS zur automatisierten Designfindung von Motoren [17], welches durch die Betrachtung der Motorbaureihe zusätzlich zur Portfolioanalyse verwendet wurde [18]. Auf diesen Ergebnissen wird mit dieser Arbeit aufgebaut.

\section{Unterstützung des Variantenmanagements von Elektromotoren durch wissensbasierte Systeme}

In der Folge wird die Unterstützung des Variantenmanagements von Elektromotoren durch wissensbasierte Systeme beschrieben. Dabei wird das bestehende KBS zur Baureihengenerierung [17] um das zweite KBS zur Konfiguration und Auslegung der WRV konzeptionell erweitert (Abschnitt 3.1). Das dafür benötigte Wissen für Auslegung der WRV wird durch die Experten bei der Produktstrukturierung der WRV gesammelt und verknüpft und entsprechend für das zweite KBS akquiriert (Abschnitt 3.2). Anschließend wird die programmseitige Umsetzung des Konzepts beschrieben (Abschnitt 3.3), gefolgt von einer abschließenden Evaluation hinsichtlich der Forschungsfrage (Abschnitt 3.4).

\subsection{Konzept und Vorgehen}

Bei dem erarbeiteten Konzept kommen zwei modulare Produktstrukturstrategien zum Einsatz: die Motor-Baureihe und der WRV-Modulbaukasten. Diese Strategien werden in zwei sequenziell arbeitenden KBS abgebildet (s. Bild 3). Basierend auf den Anforderungen legt das erste KBS gemäß [17] einen Motor aus, indem ein zweidimensionaler Motorschnitt skaliert wird. Basierend auf dem entstandenen Motor-Design wird in einem zweiten KBS unter Berücksichtigung des gewählten Schnitts und der Anforderungen die WRV konfiguriert. 


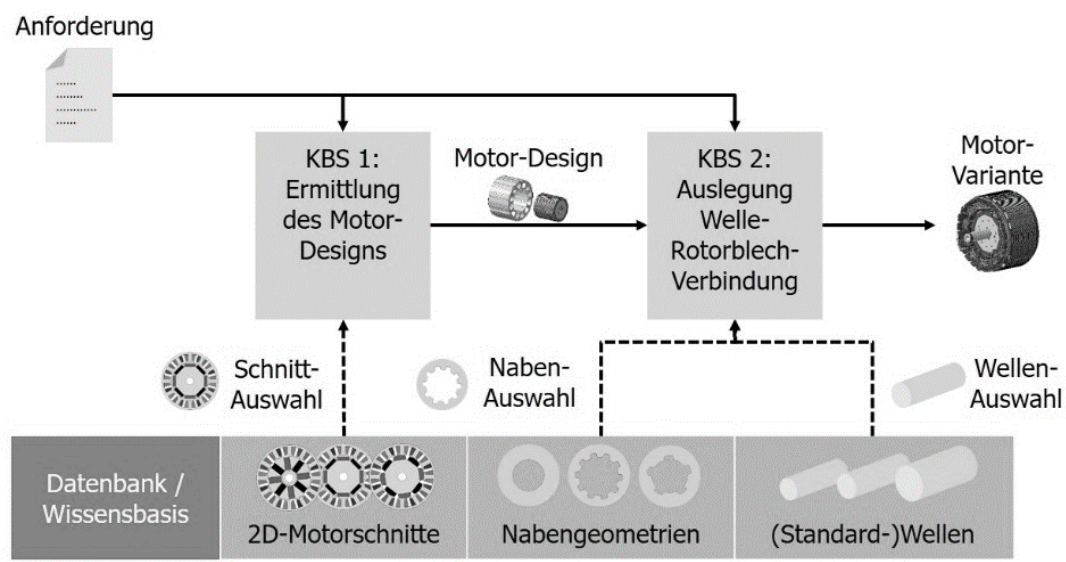

Bild 3: Konfiguration der kundenspezifischen Motorvariante mithilfe von wissensbasierten Systemen und modularen Produktstrukturstrategien

Die beiden KBS arbeiten grundsätzlich unabhängig voneinander und werden auch separat gepflegt. So stehen dem zweiten KBS verschiedene Nabengeometrien inklusive der Auslegungslogik zur Verfügung. Bei den Wellen handelt es sich aufgrund der Abtriebsgeometrie um kundenspezifische Bauteile. Eine Standardisierung ist hier nur auf die Nenndurchmesser möglich, die Freiheitsgrade der Übermaßpassung werden durch das KBS ermittelt. Die Inferenzkomponente des Programms wird in der objektorientierten Programmiersprache C\# konzipiert, während die Instanzen der Motorschnitte, Nabengeometrien sowie Wellennenndurchmesser in einer SQL-Datenbank parametrisiert abgelegt werden. Diese Trennung bietet dem Experten die Möglichkeit, jederzeit neue Instanzen hinzuzufügen. Bei den Nabengeometrien ist zudem die Einbindung der zugehörigen Berechnungslogik notwendig, die in Form von einer zusätzlichen Klasse programmiert wird. Die interne Varianz kann somit toolseitig beherrscht werden. Bei Änderungen einer Berechnungslogik oder einer Geometrie wird diese mittels einer neuen Instanz versioniert, während die auslaufende Instanz deaktiviert wird. Durch eine Wartungsansicht ist es möglich, den Reifegrad der Auslegung durch den Experten sukzessive zu erhöhen.

\subsection{Wissensakquise für die Konfiguration und Auslegung im KBS}

Wie bei herkömmlichen WNV unterliegt auch die WRV dem Coulombschen Reibschlussgesetz als physikalisches Wirkprinzip. Das Vorgehen zur Dimensionierung einer WNV ist dabei umfassend bekannt [2]. Bei der Dimensionierung der WRV von Elektromotoren wird über die Mechanik hinaus domänenspezifisches Wissen aus der Elektromagnetik und Fertigung benötigt, welches in Folge 
kurz beschrieben wird. Aus dem resultierenden Lösungsraum gilt es abschlieBend eine Variante zu erzeugen, die die Kundenanforderung erfüllt (s. Bild 4).

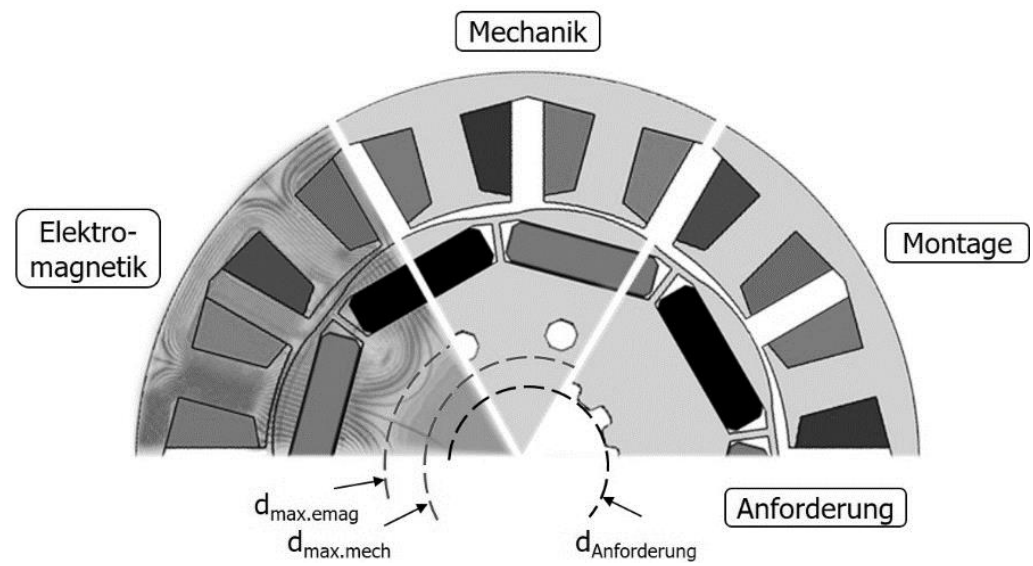

Bild 4: Schematische Darstellung der Einflussgrößen auf die Dimensionierung der Welle-Rotorblech-Verbindung

Bedingungen Elektromagnetik: Die Statorbaugruppe induziert durch die stromdurchflossene Wicklung ein elektromagnetisches Feld in die mit Permanentmagneten bestückte Rotorbaugruppe. Um die Magneten herum bildet sich, wie in Bild 4 (links) dargestellt, der elektromagnetische Fluss aus. Damit die WRV das Magnetfeld des Motors nicht negativ beeinflusst, definiert der Simulationsingenieur einen maximalen Durchmesser $d_{\text {max.emag }}$ der WRV.

Bedingungen Mechanik: Um möglichst leichte und schnell reagierende Motoren zu erhalten, wird der Rotor oftmals mit kreis- oder tropfenförmigen Aussparungen versehen. Diese sind unter der Magnetmitte angeordnet und reduzieren das Massenträgheitsmoment und Gewicht (s. Bild 4, mitte). Auch dieses Gestaltungsmerkmal beeinflusst die Auslegung der WRV, sodass der Produktentwickler einen maximalen Durchmesser $d_{\text {max.mech }}$ festlegt, falls eine Aussparung verwendet wird. Weiter erfüllt die WRV die Funktion, das geforderte Drehmoment zu übertragen. Die Auslegung der WRV erfolgt in diesem Beispiel analog zur WNV durch einen Pressverband. Durch das Übermaß in der Passung wird eine Pressung erzeugt. Abhängig von der Fügefläche kann die maximal übertragbare Kraft sowie das Drehmoment abgeleitet werden [2].

Bedingungen Fertigung und Montage: Um Wirbelstromverluste von Rotor und Stator zu reduzieren, werden diese durch einen lamellierten Aufbau in gestapelten Elektroblechen realisiert. Die detaillierte Rotorgeometrie wird im 
hier betrachteten Verfahren mittels Hubstanzen aus den Rohblechen getrennt. Hierbei kommen mehrere Trennschritte und Werkzeuge zum Einsatz. Um Prozesseinflüsse auszugleichen, werden die Bleche zueinander jeweils verdreht. Bei der Montage können in der Folge der Verdrehung und der hohen Pressung Schäden durch Verkanten oder makroskopische Schäden durch Spanbildung entstehen. Bei lamellierten Elektroblechpaketen versucht man diesem Fehlerbild durch spezielle Nabenformen entgegenzuwirken, welche einen Toleranzausgleich zulassen [19]. Je nach Anforderung wird statt einer zylindrischen Fügefläche eine verzahnte oder polygonförmige Kontur gewählt. Dabei muss aufgrund der Blechdrehung die Anzahl der Magnete (Polzahl) mit der Anzahl der "Zähne" übereinstimmen. Diese Gestaltung reduziert die in der Passung wirkende Fügefläche und somit die übertragbare Kraft und hat zudem einen Einfluss auf den Reibhaftkoeffizienten. Dieses zur Auslegung benötigte, implizit heuristische Wissen wurde bereits in der vorausgehenden Forschung mittels erweiterter, wissens- und matrixbasierter Produktbeschreibung (eWMBPB) formalisiert [20] und kann in dem wissensbasierten System berücksichtigt werden.

Bedingungen Anforderung: In der Regel wird die Welle seitens des Kunden mittels eines Durchmessers und einer Abtriebsgeometrie spezifiziert. Zudem müssen die Elektromotoren Lastzyklen fahren, wobei die maximalen Drehmomente durch die WRV übertragen werden müssen.

\subsection{Umsetzung des Variantenmanagements im KBS}

Im definierten Modulbaukasten der WRV wurden drei notwendige Freiheitsgrade identifiziert: Polzahl, Nabenform und Wellennenndurchmesser. Alle weiteren Merkmale lassen sich von diesen Parametern durch das formalisierte Wissen ableiten. Zum derzeitigen Standpunkt werden zwei verschiedene Polzahlen ( 8 und 10 ) benötigt, um eine WRV für alle Motorschnitte erzeugen zu können. Dabei stehen drei verschiedene Nabenformen und insgesamt sechs Wellennenndurchmesser zur Verfügung. Entsprechend der Variantentabelle (s. Tabelle 1) entspricht dies einer internen Varianz von 36 WRV.

Tabelle 1: Übersicht über die möglichen Varianten im KBS2

\begin{tabular}{cccc}
\hline Optionen \ Parameter & Polzahl & Nabenform & Wellennenndurchmesser \\
\hline 1 & 8 & Nabenform A & 8 \\
2 & 10 & Nabenform B & 10 \\
3 & & Nabenform C & 12 \\
4 & & & 14 \\
5 & & & 15 \\
6 & & & 20 \\
\hline Variantenzahl & $\mathbf{2}$ & $\mathbf{3}$ & $\mathbf{6}$ \\
\hline Theoretische maximale Varianz & & 36
\end{tabular}


Diese Varianz wird im fertigen KBS (s. Bild 5) abgebildet. Der Programmablauf stellt sich wie folgt dar: Zunächst lädt der User die Anforderungen (maximaler Durchmesser, Lastzyklen und Materialien) über die Schnittstelle zum Anforderungsmanagement. Anschließend kann die Baureihenvariante des Motors aus der Datenbank gewählt werden. Da die Polzahl ein Merkmal des Motorschnitts ist, wird dieser Freiheitsgrad nach Auswahl vorbelegt und es verbleiben noch 18 Freiheitsgrade bei der Konfiguration der WRV. Die Assistenzfunktion des KBS ermittelt nun durch eine Optimierung automatisch die bestmögliche Nabenform mit Wellennenndurchmesser und schlägt sie dem Nutzer vor.

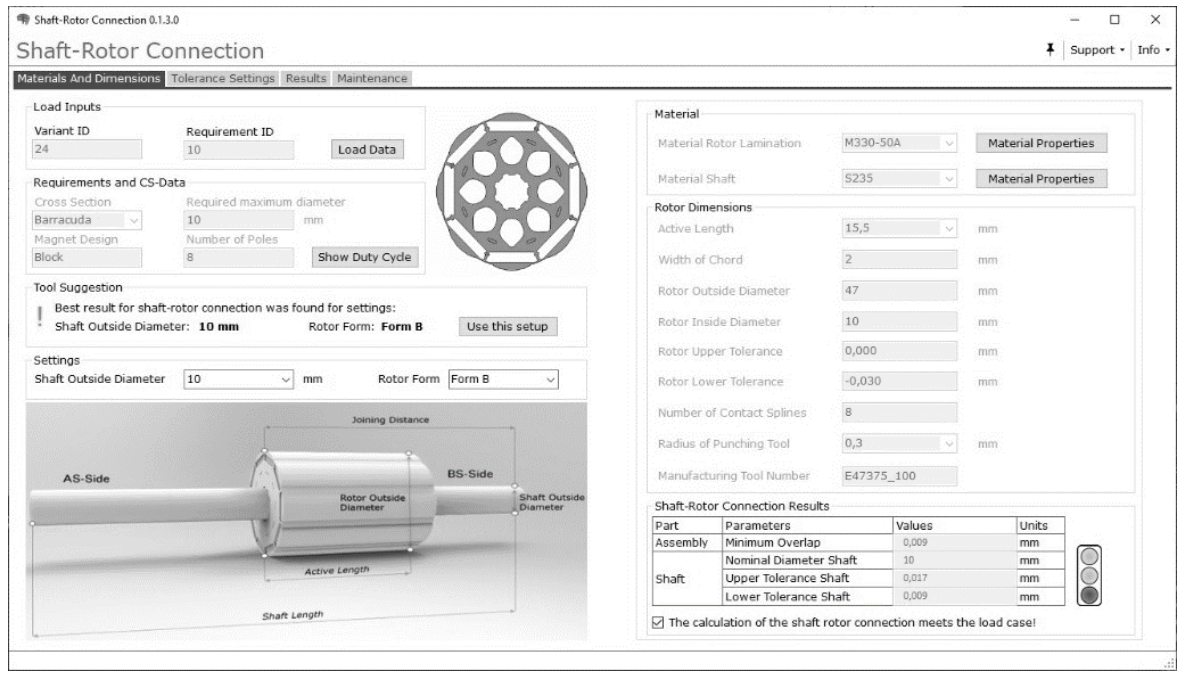

Bild 5: Hauptansicht des User-Interfaces für die wissensbasierte Konfiguration der Rotorgeometrie und wissensbasierte Auslegung der Wellengeometrie

Dabei sind vor allem Lastfall, Rotorpaketlänge, Reibhaftkoeffizient, Durchmesser und Nabenform entscheidend. Möchte der Nutzer den Vorschlag nicht übernehmen, kann er den Durchmesser und die Nabenform darüber hinaus selbst definieren. Output des Tools sind detaillierte Informationen über die ausgewählte Nabengeometrie sowie die geometrischen Abmaße und Toleranzen, mit der die Welle gefertigt werden muss. Für die Nabengeometrie wird außerdem - sofern vorhanden - die Werkzeugnummer ausgegeben.

\subsection{Ergebnisse und Diskussion}

Ziel dieser Arbeit war es, die interne Variantenvielfalt und die internen Aufwände bei der Entwicklung von Elektromotoren zu reduzieren und zu beherr- 
schen. Mit dem beschriebenen Konzept basierend auf modularen Produktstrukturstrategien lässt sich die interne, rotorseitige Varianz der WRV auf 36 festlegen. Durch die wissensbasierte Unterstützung ist eine manuelle Auslegung der WRV nicht mehr notwendig, da das KBS automatisch die Freiheitsgrade belegt und eine Lösung auf Knopfdruck empfiehlt. Bringt man die WRV-Varianz in Zusammenhang mit dem ersten KBS für die Schnittauslegung so erhöht sich die externe Varianz weiter. Bei derzeit 10 Motorschnitten und den verbleibenden 18 Freiheitsgraden (durch die vorbelegte Polzahl) entstehen insgesamt 180 theoretische 2D-Motorvarianten. Durch eine zusätzliche, wissensbasierte Eingrenzung des maximalen Durchmessers entsprechend den genannten EinflussgröBen aus Elektromagnetik und Mechanik verbleiben noch 50 2D-Varianten, welche insgesamt durch 17 Werkzeuge abgebildet werden können (s. Bild 6). Da sich diese Werkzeuge auf die Fertigung eines Rotorblechs beziehen, beeinflusst der Modulbaukasten der WRV die 3D-Ergebnisse der Motorschnitt-Baureihe nicht. Die absolute externe Varianz der dreidimensionalen Motoren ist daher direkt abhängig von der Anzahl der Motorschnitte sowie der Grenzen der Freiheitsgrade Länge und Windung.

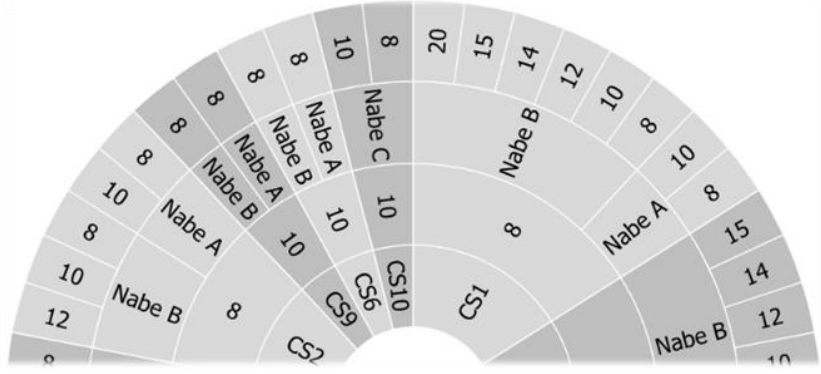

Bild 6: Auszug der 50 WRV-Varianten für die bestehenden 10 Motorschnitte

Mit dem vorgelegten Konzept ist es möglich, Nabengeometrie und Wellennenndurchmesser standardisiert zu konfigurieren. Eine Standardisierung der Welle hinsichtlich der Passung ist aber weiterhin nicht sinnvoll. So lassen sich zwar mehrere Projekte mit der gleichen Passung realisieren, aber die Abtriebsgeometrie bleibt eine kundenspezifische Schnittstelle, weswegen auch die Welle als Bauteil weiterhin als Individualteil gehandhabt wird.

\section{Zusammenfassung und Ausblick}

Mit diesem Beitrag wurde die Unterstützung des Variantenmanagements von Elektromotoren durch wissensbasierte Systeme untersucht und anhand der Welle-Rotorblech-Verbindung evaluiert. Im vorgestellten Konzept kommen zwei kombinierte wissensbasierte Systeme zum Einsatz, welche die Regeln und das 
Wissen über die Konfiguration innerhalb der modularen Produktstrukturen Baureihe und Modulbaukasten beinhalten. Dadurch wird dem Entwickler bei der Konfiguration und Auslegung des Elektromotors assistiert und die manuellen Aufwände sowie Fehler werden stark reduziert. Zudem war es durch die definierten Freiheitsgrade möglich, die interne Varianz zu reduzieren und festzulegen. In der weiteren Forschung sollen die verbleibenden Bauteile wie Gehäuse und Kugellager ebenfalls in das Variantenmanagement integriert werden.

\section{Literatur}

[1] Hendershot, J. R. und Miller, T. J. E.: „Design of Brushless PermanentMagnet Motors", Magna Pysics Pub. Clarendon Press, Hillsboro, 1994.

[2] Kollmann, F. G.: „Welle-Nabe-Verbindungen”, Springer, Berlin, 1984.

[3] Heina, J.: „Variantenmanagement”, Springer, Wiesbaden, 1999.

[4] Krause, D. und Gebhardt, N.: „Methodische Entwicklung modularer Produktfamilien", Springer Vieweg, Berlin, 2018.

[5] Meyer, M. H. und Lehnerd, A. P.: "The Power of Product Platforms”, The Free Press, New York, 1997.

[6] Ulrich, K.: "The Role of Product Architecture in the Manufacturing Firm", Research Policy, Vol. 24, 1995, S. 419-440.

[7] Roach, G. M. und Cox, J. J.: "A Case Study of the Product Design Generator", in: Simpson, T. W., Siddique, Z. und Jiao, J. R. (Hrsg.): „Product Platform and Product Family Design", Springer, New York, 2006, S. 499512.

[8] Ponn, J.: „Portfolio Management for Electric Drives in Powertools at Hilti: Challenges and Solution Approaches", Proceedings of ICED 2015, Weber, C. et al. (Hrsg.), The Design Society, Milan, 2015, S. 105-114.

[9] Eppler, M. J., Seifried, P. und Röpnack, A.: „Improving Knowledge Intensive Processes through an Enterprise Knowledge Medium", Proceedings of the 1999 ACM SIGCPR Conference, Prasad, J. (Hrsg.), Gabler 1999, S. 222-230.

[10] VDI 5610 Blatt 1: „Wissensmanagement im Ingenieurwesen", Beuth, Berlin, 2009. 
[11] Nonaka, I. und Takeuchi, H.: "The Knowledge-Creating Company", Oxford University Press, New York, 1995.

[12] La Rocca, G.: „Knowledge Based Engineering: Between AI and CAD: Review of a Language Based Technology to Support Engineering Design", Advanced Engineering Informatics, Vol. 26, 2012, S. 159-179.

[13] Styczynski, Z. A., Rudion, K. und Naumann, A.: „Einführung in Expertensysteme, Springer Vieweg, Berlin, 2017.

[14] Favi, C. et al.: „An Integrated Approach and It Platform to Optimise Electric Motor Engineering and Design", Int. J. Information Technology and Management, Vol. 13, 2014, S. 134-153.

[15] Karnavas, Y. L. und Chasiotis, I. D.: „A Simple Knowledge Base Software Architecture for Industrial Electrical Machine Design: Application to Electric Vehicle's In-Wheel Motor, Proceedings of ISAT 2015, Wilimowska, Z. et al. (Hrsg.), Springer, Cham, 2016, S. 111-122.

[16] Rivera, C. A. et al.: „A Knowledge Based System Architecture to Manage and Automate the Electrical Machine Design Process", IEEE Int. Workshop of Electronics, Control, Measurement, Signals and their Application to Mechatronics (ECMSM), IEEE, Donostia-San Sebastian, 2017.

[17] Tüchsen, J. et al.: „Data Driven Design Selection and Generation - an Industrial Case Study on Electric Motors", Proceedings of DESIGN 2018, The Design Society, Marjanović, D. et al. (Hrsg.), Dubrovnik, 2018, S. 1709-1720.

[18] Tüchsen, J. et al.: „Data Driven Product Portfolio Analysis of Electric Motors Based on Product Platforms Using Knowledge-Based Systems", Proceedings of ICED 2019, The Design Society, Delft, 2019, S. 2537-2546.

[19] Tong, W.: „Mechanical design of electric motors ", CRC Press, Boca Raton, 2014.

[20] Tüchsen, J. et al.: "Methode zur Einbindung von heuristischem Wissen in die erweiterte matrixbasierte Produktbeschreibung", 16. Gemeinsames Kolloquium Konstruktionstechnik, Rieg, F. et al. (Hrsg.), Bayreuth, 2018, S. 337-348. 